\title{
Blood-derived rejuvenation
}

Studies have shown that blood from young mice, when introduced into old animals, counteracts ageingrelated effects in various tissues, including the brain. Building on these findings, three new studies show that young blood improves brain and muscle function in aged mice, and provide insight into the mechanisms underlying these effects.

Wyss-Coray and colleagues examined the effects of young blood on the hippocampus (which shows ageing-related functional decline) in part by surgically joining the circulatory systems of two mice (a technique called parabiosis).

Compared with old isochronic parabionts (old mice that had been surgically connected to other old mice), old heterochronic parabionts (old mice that had been surgically connected to young mice) exhibited increased expression of immediateearly genes in dentate gyrus (DG) cells and a rise in phosphorylated CREB1 (cyclic AMP-responsive element-binding protein 1) in the DG and CA1 - findings consistent with increased synaptic plasticity. Indeed, hippocampal slice recordings showed enhanced long-term potentiation in the DG of old heterochronic parabionts.

In agreement with these findings, old mice that received repeated injections of plasma from young animals exhibited increased learning and memory in hippocampus-dependent behavioural tasks compared with old mice that received injections of old plasma. Interestingly, expressing a dominant-negative form of CREB1 into the DG of old mice partially reduced the cognitive-enhancing effects of young plasma. This suggests that increased CREB1 signalling in part mediates the effect of young plasma on hippocampus-dependent cognitive function in old mice.

Brain ageing is also associated with decreased adult neurogenesis, possibly resulting from a reduction in the number of adult neural stem cells, and a previous study showed that old heterochronic parabionic mice generated more neurons in the subgranular zone of the DG, a region of adult neurogenesis, than old isochronic parabionts.

Rubin and colleagues found that old heterochronic parabionic mice have increased numbers of neural stem cells in the subventricular zone (SVZ) of the lateral ventricles, another site of adult neurogenesis. Neuroblasts derived from SVZ-born stem cells migrate to the olfactory bulb, and bromodeoxyuridine staining revealed that old heterochronic parabionts had nearly double the number of newborn olfactory neurons than did old isochronic parabionts. Interestingly, old heterochronic parabionts performed better in an odour discrimination task, suggesting that exposure to young blood improves olfactory function in aged mice by promoting SVZ neurogenesis.

Three-dimensional reconstructions of brain vasculature revealed that old heterochronic parabionts had greater blood vessel volume in the SVZ and other regions than old isochronic parabionts. Moreover, daily injections of growth/differentiation factor 11 (GDF11) - which is found in serum and known to counteract ageing-related effects on cardiac muscle - increased blood vessel volume and SVZ stem cell number in old mice, suggesting that GDF11-induced vasculature remodelling may at least in part mediate the increased adult

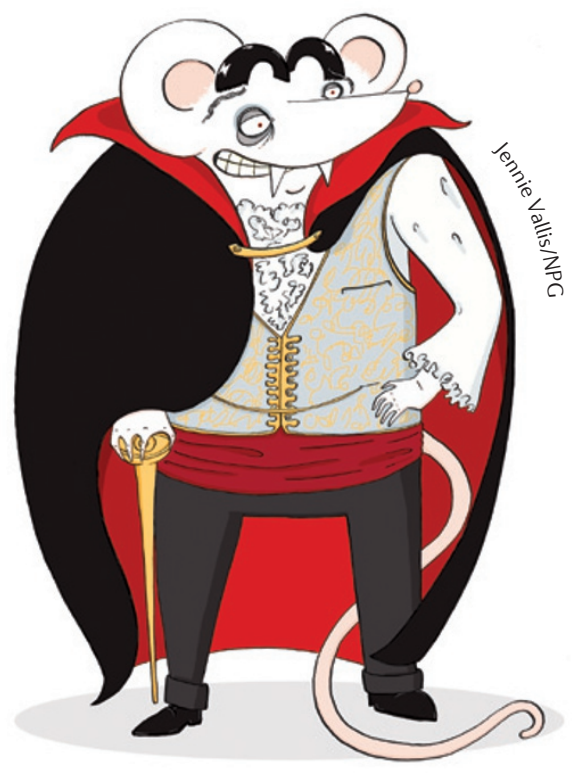
neurogenesis and olfactory function in old heterochronic parabionts.

The ability of GDF11 to reverse ageing-associated tissue dysfunction was further highlighted by a recent study on muscle cells. Aged muscle exhibits a decrease in the number and function of muscle stem cells, and Wagers and colleagues showed that treatment of aged mice with recombinant GDF11 increased the number of these cells and their activity in regeneration assays, and enhanced muscle function (as measured by grip strength and endurance exercise paradigms).

Together, these studies provide further evidence for the potentially rejuvenating effects of young blood on ageing tissues. Specifically, they reveal two different potential pathways by which circulatory factors may restore function in aged brains.

Darran Yates

ORIGINAL RESEARCH PAPERS Villeda, S. A. et al. Young blood reverses age-related impairments in cognitive function and synaptic plasticity in mice. Nature Med. http://dx.doi. org/10.1038/nm.3569 (2014)|Katsimpardi, L. et al. Vascular and neurogenic rejuvenation of the aging mouse brain by young systemic factors. Science 344, 630-634 (2014) | Sinha, M. et al. Restoring systemic GDF11 levels reverses age-related dysfunction in mouse skeletal muscle. Science $\mathbf{3 4 4}$, 649-652 (2014)

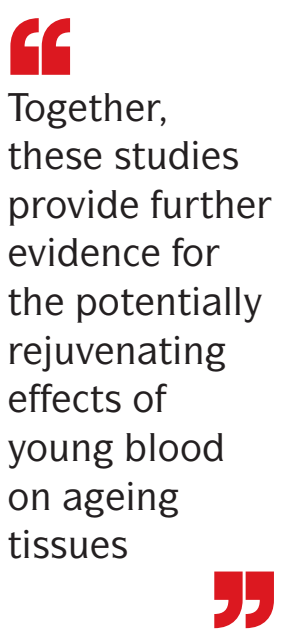

